\title{
ANÁLISIS DE LAS OPINIONES DE UN GRUPO DE ESTUDIANTES UNIVERSITARIOS SOBRE LOS FACTORES QUE LES IMPULSARON A ELEGIR SUS ESTUDIOS
}

\author{
Rakel Varela Ona \\ Departamento de Geografía, Prehistoria y Arqueología \\ Universidad del País Vasco-Euskal Herriko Unibertsitatea (UPV-EHU) \\ rakel.varelahu.es \\ Maria Teresa Vizcarra Morales \\ Departamento de Didáctica de la Expresión Musical, Plástica y Corporal \\ Universidad del País Vasco-Euskal Herriko Unibertsitatea (UPV-EHU) \\ mariate.bizkarra@ehu.es \\ Pello Urkidi Elorrieta \\ Departamento de Didáctica de las Ciencias Sociales \\ Universidad del País Vasco-Euskal Herriko Unibertsitatea (UPV-EHU) \\ pello.urkidi@ehu.es
}

\section{RESUMEN}

Esta investigación pretende conocer las razones que llevan al alumnado a escoger la titulación de Geografía y Ordenación del Territorio en la UPV/EHU. Para ello, han sido entrevistados los estudiantes de los cuatro cursos de Geografía y se ha indagado en los recuerdos que el alumnado tiene de sus estudios previos. Así mimo, se desea conocer en qué áreas de estudio se encuentran más cómodos o qué materias han recibido antes de acceder a la Universidad que se relacionen con el Grado escogido. Mediante las respuestas obtenidas se ha delimitado una definición de la ciencia geográfica desde la percepción del alumnado, para saber así cuál es la representación social que tienen de esta ciencia. Esta definición va a venir construida a través de las ideas previas con las que acceden a la Universidad.

Palabras clave: Estudios de Grado, Geografía, ideas previas, representaciones sociales.

Fecha de recepción: abril 2014.

Fecha de aceptación: mayo 2015. 


\section{ABSTRACT}

The paper presented aims to determine the reasons that lead the students to choose the degree of Geography and Regional Planning. For this the students of the four courses in Geography have been interviewed and has explored the memories that students have of their previous studies as well as in knowing what areas of study they are most comfortable with or what matter they have received before accessing to the University related to the degree chosen. Through the answers has tried to delimit a definition of chores in the geography from the point of view of the student, to know what the social representation of science built across the previous ideas that have when they access to the University.

Keywords: Degree studies, Geography, previous ideas, social representations.

\section{INTRODUCCIÓN}

En los últimos años son numerosos los cambios acontecidos en el marco de la Educación Superior. En el caso concreto que nos atañe, estos cambios han quedan reflejados en las asignaturas a impartir o en el contenido de dichas asignaturas, incluso ha supuesto un cambio en el nombre de la propia titulación, pasando de Licenciatura en Geografía a Grado en Geografía y Ordenación del Territorio (Libro Blanco de Geografía, 2005; Tulla, 2010). Un elemento novedoso de los Grados ha sido la adscripción de la titulación, por parte de muchos departamentos, a la rama de Ciencias Sociales y Jurídicas, cuando tradicionalmente se había adscrito a la rama de Arte y Humanidades (Esparcia y Sánchez, 2012).

Al ser un proceso reciente, ya que en el curso 2013-14 termina la primera promoción de Grado, es interesante valorar la opinión del alumnado con el fin de poder detectar si hay algún aspecto mejorable, de cara a una futura revisión del mismo. Más aún, teniendo en cuenta la situación de crisis económica en la que vivimos, proporcionar una educación de calidad y con una buena perspectiva laboral, puede ser decisivo para la supervivencia de este Grado. Por eso, éste es un trabajo de investigación exploratorio para conocer aquellos aspectos de mejora que puedan detectarse en el Grado de Geografía y Ordenación del Territorio.

Uno de los pilares de la base teórica de este estudio es el Libro Blanco de Geografía y Ordenación del Territorio (ANECA 2005) que fue diseñado para guiar a las Universidades a transformar sus Licenciaturas en Grados. Las exigencias del Espacio Europeo de Educación Superior han supuesto un estudio en profundidad del estado de la Geografía en Europa y no solo desde el punto de vista académico, sino también sobre las posibilidades de inserción laboral de los egresados, y el grado de actualización a las nuevas demandas de la sociedad.

Si se revisan los estudios más recientes, podemos observar que el alumnado que accede a los grados de Geografía no sabe con exactitud lo que se va a encontrar, como así lo demuestra el análisis realizado por la Universidad de Sevilla con estudiantes de primero de la titulación de Geografía y Gestión del Territorio (Garrido, Galindo, García, y López, 2011). El objetivo principal de dicho estudio fue evaluar las causas del escaso poder de atracción de la Geografía por parte de los nuevos universitarios. Como resultados principales constata el carácter vocacional de su elección y el desconocimiento por parte del 
alumnado de lo que realmente se van a encontrar en los estudios de Geografía, a pesar del esfuerzo que hacen las Universidades por dar a conocer sus Grados mediante Jornadas de Puertas Abiertas, Ferias, etc.

La ciencia geográfica, según Marrón (2011), tiene un gran potencial educativo, porque permite conseguir aprendizajes útiles para la vida cotidiana, y para entender el mundo y los procesos que en él acontecen a distintas escalas territoriales y sociales. Partiendo de esta idea, es importante orientar al alumnado sobre el verdadero objetivo de la Geografía, que va mucho más allá de una ciencia meramente memorística.

En el estudio que llevó a cabo Marrón (2008) en la Universidad Complutense de Madrid con sus estudiantes de Magisterio (futuros profesionales de la docencia) mediante una investigación participante pretendió detectar el nivel de formación geográfica del alumnado al llegar a la universidad, no obteniendo unos resultados muy esperanzadores ya que el $76 \%$ demostró tener un conocimiento muy deficiente de los contenidos geográficos que, de acuerdo con los actuales planes de estudio, debería dominar al terminar sus estudios previos. El 15\% mostró un conocimiento medianamente aceptable de dichos contenidos y sólo el 9\% demostró haber alcanzado un adecuado conocimiento.

Otro trabajo a tener en cuenta es el de García (2008), donde se analizaron las expectativas que el alumnado tenía ante determinadas asignaturas, a fin de proporcionar al profesorado información que le permitiese conducir de forma óptima el programa de las mismas. El Colegio de Geógrafos publicó en 2008 un estudio acerca de los mencionados perfiles profesionales de los geógrafos españoles. En contra de lo que popularmente se cree, un geógrafo sirve para algo más que para la enseñanza, como así lo demuestra este estudio donde las principales ocupaciones son con un $28 \%$ asuntos de Planificación Territorial y Urbanística, con un 22\% Tecnologías de Información Geográfica y un 19\% trabajos relacionados con temas de Medio Ambiente. Quedando la enseñanza relegada a puestos finales de la clasificación obtenida.

No siempre el Geógrafo ha estado tan valorado profesionalmente. Es necesario analizar la trayectoria de la ciencia geográfica para comprender las propuestas de reforma presentadas ante el nuevo Marco de Educación Superior, con el fin de modernizar la imagen que se tiene de esta ciencia.

Es difícil definir con total precisión los límites y los contenidos de la Geografía, y éste es un problema que acompaña a esta disciplina desde sus comienzos. En sus orígenes la Geografía fue una ciencia dedicada a la descripción y representación cartográfica de la tierra, labores de gran importancia al servicio de la exploración, los descubrimientos y la colonización. Era, por tanto, una Geografía utilitarista, en relación a objetivos militares y comerciales (Plans, 1984; Méndez y Molinero, 1991). A partir de la llamada Edad de Oro de la exploración, entre los siglos XV al XIX (Holt-Jensen, 1992) se produce la transición no poco compleja de la Geografía precientífica, a la disciplina científica.

Como recogen Capel (1998) y Gómez (1998), Humbolt con su obra «Cosmos. Ensayo de una descripción física del Mundo y Ritter en «La organización del Espacio en la superficie del Globo y su función en el desarrollo histórico», pueden considerarse como los precursores de esa transición, al impulsar y reorientar el objeto de la Geografía, proporcionando la base teórica de la Geografía Científica Moderna. En este momento la brecha existente entre la Geografía Física y la Geografía Humana se acentúa. 
La evolución histórica de esta ciencia permite apreciar tres cuestiones fundamentales que se plantean como foco de discusión: la determinación del objeto específico de la Geografía, la aplicación de un método científico a los estudios de esta disciplina y la discusión acerca de la unidad de la propia Geografía (Capel, 1981,1998; Estébanez, 1982; Johnston y Claval, 1986; Unwin, 1995).

En la superficie terrestre ocurren fenómenos derivados de la dinámica de los componentes naturales del medio, y otros, que son consecuencia de la actividad antrópica, lo que permite diferenciar una Geografía Física de una Geografía Humana. Algunos autores hacen hincapié en que la separación, cada vez mayor, de ambas ramas ha provocado una desorientación social respecto a que se dedican los geógrafos (Stoddart, 1987).

A todo esto tenemos que añadir que nuestra ciencia se asocia generalmente a conocimiento enciclopédico (nombres de ciudades, altitudes de montes, ríos, etc.), elaboración de mapas y descripción de viajes. Este hecho se deriva del escaso peso de la Geografía en los planes de estudio de la enseñanza básica y secundaria, su dedicación preferente a la memorización de lugares y de sus características más tópicas. Este panorama no es propio únicamente de nuestro país, sino que resulta bastante generalizado y generalizable (Holt-Jensen, 1992).

Por todo lo comentado hasta ahora y por la necesidad de adaptarse a las nuevas demandas y a los avances tecnológicos que han obligado a la Geografía a crear una definición competitiva, hoy en día podemos definir la Geografía como la ciencia que estudia- y no solo describe- las variaciones y distribuciones de los fenómenos de la superficie terrestre -abióticos, bióticos y culturales- y sus relaciones. La ciencia geográfica intenta, de esta manera, convertirse en una intérprete global de la interacción entre el ser humano y el territorio, lo cual constituye su objeto básico. Este objeto de estudio ha originado que conceptos, como Ordenación del Territorio o Medio Ambiente, tengan un peso muy importante en los planteamientos geográficos actuales.

Esta visión de la Geografía como «Ciencia del Territorio» ha tenido un peso importante en el diseño de los nuevos grados. Con éste y otros cambios, la Universidad trata de acercar y dar a conocer la Geografía a la sociedad, para que así se pueda valorar el trabajo poco conocido que realizan los geógrafos. Para diseñar un grado habría que tener en cuenta las tendencias de índole socio-cultural, político-institucional, epistemológica-disciplinar y de las prácticas de enseñanza (Fernández y Gurevich, 2010). Es necesario analizar las ideas previas con las que el alumnado accede a esta titulación, para poder explicar el conocimiento que la población tiene respecto a esta ciencia. Se denominan ideas previas a las concepciones que tienen los estudiantes sobre diferentes fenómenos, aún sin recibir ninguna enseñanza sistemática al respecto; estas ideas se crean a partir de las experiencias cotidianas, las actividades físicas, las conversaciones con otras personas, y de la información de los medios de comunicación, entre otros factores; representan modelos coherentes de conocimiento, aunque pueden parecer incoherentes a la luz de la ciencia, o del conocimiento escolar (Driver, 1996).

Por lo tanto, son explicaciones que los estudiantes van construyendo mediante la interacción con su medio natural y social. Las características de las ideas previas son las siguientes: presentan una coherencia interna, son personales, son comunes a estudiantes de determinadas edades, y culturas, son persistentes y no se modifican fácilmente, se construyen a partir 
de la interacción con el medio, se fundamentan principalmente en las experiencias de la vida cotidiana y pueden ser un obstáculo en la comprensión del conocimiento (Rayas, 2004).

Existen más de 30 acepciones para denominar a las ideas previas recogemos de Cubero (1994) algunas de ellas: «ideas intuitivas, ciencia de los niños, representaciones de los alumnos (Osborne, Bell y Gilbert, 1983), Errores conceptuales de Helm (1980), Preconcepciones de Novak (1977), Concepciones alternativas, o marcos alternativos de Driver y Easley (1982) que después denominaron ideas de los niños; Razonamiento espontáneo de Viennot (1979). Representaciones de Giordan (1982), preconceptos, de Mc Dremott (1984), y Duit (1984)».

Muy relacionado con el término de ideas previas están las representaciones sociales del alumnado. En la década de los sesenta Moscovici (recogido en Moliner 2001) acuña la «teoría de las Representaciones Sociales» que constituye una de las aportaciones cognoscitivas más originales en el ámbito de la psicología social. La base teórica de las representaciones sociales hace referencia a cómo los individuos construyen, organizan y comparten los conocimientos dentro de la sociedad: una representación consiste, entonces, en un sistema de valores, conocimientos y prácticas que permite a las personas no solo orientarse dentro de su ambiente social, sino también, dar un sentido a los acontecimientos.

Las representaciones sociales, en calidad de sistemas de interpretación que sostienen nuestras relaciones con el mundo y con los demás, orientan y organizan el comportamiento y la comunicación. Promueven también la difusión y la asimilación de los conocimientos, el desarrollo tanto individual, como colectivo, la definición de las identidades individuales y de grupo, las expresiones de los grupos y las transformaciones sociales (Jodelet, 1986).

La génesis, la evolución y el cambio de las representaciones sociales se determinan de hecho por los procesos de comunicación social, las interacciones entre las personas y entre los grupos, los intercambios lingüísticos y culturales, el flujo continuo de conocimientos, opiniones y emociones que caracteriza la dinámica social (Deitinger, et al. 2009).

Por lo que podemos considerar la representación social, en palabras de Gutiérrez (1998), como la «teoría» mediante la que personas y grupos obtienen una lectura de la realidad y, además, toman una determinada posición en relación a ella.

En nuestro estudio, la realidad que queremos delimitar es la realidad de la ciencia geográfica donde las ideas previas nos llevan a construir representaciones sociales alejadas del verdadero quehacer geográfico. Estas ideas, bien por la formación que las generaciones anteriores recibieron, bien por la propaganda escasa que se ha hecho o bien por otros motivos, pueden considerarse obsoletas y pertenecientes a épocas anteriores donde se relacionaba a la geografía con cuestiones memorísticas, de interpretación de mapas o asuntos climáticos. Por todo lo anterior, la ciencia Geográfica está limitada por la representación social que la sociedad tiene asumida de esta disciplina, y el sistema educativo y la universidad son los posibles agentes para repensar los constructos sobre los que se asientan las representaciones sociales.

El aprendizaje científico y el desarrollo cognitivo que éste implica, pueden concebirse, al menos en parte, como un proceso de cambio de las teorías personales implícitas por otras teorías explícitas y científicas (Dibarboure, 2007). Esta labor, como ya hemos comentado, debería ser realizada por la Universidad, de ahí, que para un buen diseño de las enseñanzas del Grado es importante conocer las ideas previas y las representaciones sociales con las 
que el alumnado ingresa en la Universidad. Realizar un buen diseño supone, además, dotar al alumnado de una definida identidad profesional, es decir, que la definición que la persona hace de sí misma en relación con un espacio de trabajo y un colectivo profesional u ocupacional de referencia, sea claro y dinámico (Balduzzi y Corrado, 2010).

Dentro de las variadas ramas que alberga la Geografía podemos encontrar trabajos basados en las representaciones sociales, como por ejemplo el artículo de Larsen, Foulkes, Sorenson y Thompson (2011) acerca de la importancia que tienen las representaciones sociales en el mundo rural a la hora de llevar a cabo políticas de mejora medio ambientales. O los trabajos de Halfacree $(1993,1995,1998)$ o Camarero y Oliva $(2002,2008)$ donde pretenden explicar la mercantilización de la rusticidad como signo de nuevo culto a los elementos naturales; la calidad de vida se asocia con los «encantos rústicos»: paisajes rurales, vivir en la naturaleza, comida tradicional... El turismo revaloriza los paisajes como objeto de mejora. Es decir, la representación social de lo rural es utilizada en la vida diaria distorsionada e idealizada.

Centrándonos en los estudios de Geografía General podemos observar que no es precisamente idílica la imagen que se vende de esta ciencia. En un estudio realizado por Marrón (2011) se pretende detectar el grado de interés que para los estudiantes de magisterio de la Universidad Complutense de Madrid tiene el estudio de la Geografía para su educación como ciudadanos de la sociedad actual. Se les preguntó qué valor le concedían a la Geografía dentro de las disciplinas académicas y los resultados fueron poco halagüeños, pues únicamente el $32 \%$ de los estudiantes manifestó una actitud favorable hacia la Geografía como disciplina académica. Por el contrario, el $68 \%$ restante la percibió negativamente y la consideró una asignatura carente de interés y poco útil, manifestando que la mayor parte de los contenidos que abordan en ella los olvidaban en poco tiempo.

$\mathrm{Al}$ indagar acerca de las experiencias que habían vivido en relación con la enseñanza aprendizaje de esta ciencia, la inmensa mayoría (77\%), manifestó haberla estudiado de forma memorística exclusivamente.

Es por ello, que tradicionalmente la Geografía (Esparcia y Sánchez, 2012) ha sido refugio de segundas, terceras y hasta cuartas opciones para los estudiantes. El estudiante que suele ingresar en Geografía no solo no la tiene entre sus preferencias, sino que no tiene una idea precisa de en qué consiste y qué se va a encontrar, y todo tiene como consecuencia una actitud, inicial al menos, poco receptiva. Resultados similares obtuvo Miranda (2006) en su análisis de obstáculos para la enseñanza de la Geografía, ya que en su caso, la mayoría de los estudiantes no consideraba la Geografía como una ciencia, sino como una materia o asignatura relacionada con el relieve, el clima o los mapas.

La importancia de descubrir el contenido de la representaciones sociales permitirá comprender cómo se dan los cambios con respecto a la Geografía (Yépez, 2010; Aisenson et al., 2004).

Tras analizar las experiencias expuestas por los estudiantes los objetivos de esta investigación son conocer las ideas previas que sobre los estudios de geografía tiene el alumnado del Grado en Geografía y Ordenación del Territorio y de la Licenciatura de Geografía; extraer de las definiciones de los propios estudiantes su concepción, su definición propia de la Geografía; determinar en qué medida la elección de los estudios de la titulación de Geografía es vocacional, y descubrir sus expectativas vocacionales. 


\section{MÉTODO}

Para conocer las razones que han llevado al alumnado a escoger la carrera de Geografía se elaboró un cuestionario compuesto de preguntas de respuesta abierta y cerrada que se pasó a todo el alumnado matriculado en Geografía durante el curso 2011-12 y al alumnado matriculado en primero durante el curso 2012-13. Para analizar la información recogida se realizó un análisis de contenido, que dio origen a un Sistema Categorial (véase Figura 1) en el que las categorías aparecen ordenadas jerárquicamente en función del significado y no por el número de respuestas obtenidas. El Sistema Categorial nos sirve para ordenar el informe de resultados.

\begin{tabular}{|c|c|c|}
\hline \multirow{8}{*}{$\begin{array}{c}\text { Razones: } \\
\text { argumentaciones } \\
\text { que utilizan } \\
\text { para justificar la } \\
\text { elección de sus } \\
\text { estudios (p.12). } \\
\text { (117) }\end{array}$} & \multirow[t]{3}{*}{$\begin{array}{l}\text { Razones de índole personal (RPS). } \\
\text { (58) }\end{array}$} & $\begin{array}{l}\text { Relacionado con sus aficiones (Naturaleza, } \\
\text { Cultura, Viajes, mapas...)(10) }\end{array}$ \\
\hline & & $\begin{array}{l}\text { Inquietud e interés por aprender y } \\
\text { disponibilidad de tiempo para hacerlo. (12) }\end{array}$ \\
\hline & & Gusto por la Geografía. (36) \\
\hline & Razones profesionales (RProf).(5) & Acceso al mercado laboral. (5) \\
\hline & \multirow[t]{3}{*}{ Razones académicas (RA). (52) } & Nota de selectividad. (18) \\
\hline & & $\begin{array}{l}\text { Experiencia relacionada con experiencias } \\
\text { escolares previas (Materia o profesor). (13) }\end{array}$ \\
\hline & & Contenido de la carrera. (21) \\
\hline & $\begin{array}{l}\text { Razones relacionadas con su } \\
\text { entorno social (ES). (2) }\end{array}$ & Entorno familiar(2) \\
\hline \multirow{7}{*}{$\begin{array}{l}\text { Ideas previas } \\
\text { y expectativas } \\
\text { relacionadas } \\
\text { con los Estudios } \\
\text { geográficos. (p. } \\
13 \text { y 25). (152) }\end{array}$} & Lo que esperaba (29) & \\
\hline & No esperaba nada en especial(5) & \\
\hline & Adjetivo positivo (11)(neg 1) & \\
\hline & Continuación del bachiller (2) & \\
\hline & $\begin{array}{l}\text { Respuestas relacionadas con otras } \\
\text { áreas (24) }\end{array}$ & \\
\hline & $\begin{array}{l}\text { Relaciones con las áreas } \\
\text { geográficas(45) }\end{array}$ & $(* * *)(\mathrm{p} .13)$ \\
\hline & $\begin{array}{l}\text { Áreas geográficas recibidas en el } \\
\text { instituto (p.25) (76) }\end{array}$ & $(\mathrm{p} .25)$ \\
\hline \multirow{2}{*}{$\begin{array}{l}\text { ¿Qué entienden } \\
\text { por } \mathrm{G}^{\mathrm{a}} \text { hoy en } \\
\text { día? (p.19) (116) }\end{array}$} & Arma política. (1) & \\
\hline & $\begin{array}{l}\text { Búsqueda de respuestas de la } \\
\text { realidad. (1) }\end{array}$ & \\
\hline
\end{tabular}




\begin{tabular}{|c|c|c|}
\hline \multirow{14}{*}{$\begin{array}{l}\text { ¿Qué entienden } \\
\text { por } G^{a} \text { hoy en } \\
\text { día? (p.19) (116) }\end{array}$} & \multirow{6}{*}{$\begin{array}{l}\text { Estudio de la tierra/PLANETA } \\
\text { (61) }\end{array}$} & Planeta (23) \\
\hline & & Evolución. (3) \\
\hline & & Parte física. (2) \\
\hline & & $\begin{array}{l}\text { Interrelaciones de los elementos del } \\
\text { territorio. (16) }\end{array}$ \\
\hline & & Interpretación. (5) \\
\hline & & Estudio integral del territorio (36) \\
\hline & \multirow{8}{*}{$\begin{array}{l}\text { Conceptos relacionados con la } \\
\text { definición como CIENCIA (30) }\end{array}$} & Ciencia (2) \\
\hline & & Ciencia social. (5) \\
\hline & & Indefinida. (2) \\
\hline & & Multidisciplinar. (11) \\
\hline & & Necesaria. (2) \\
\hline & & Analítica. (4) \\
\hline & & Descriptiva. (3) \\
\hline & & Resolución de problemas. (1) \\
\hline \multirow{2}{*}{$\begin{array}{c}\text { Características } \\
\text { asociadas a la } \\
\text { Geografía p.20. } \\
\text { (880) }\end{array}$} & $\begin{array}{l}\text { Definición por conceptos }(* * *) \\
\text { p.20 }(516 \text { de } 585)\end{array}$ & \\
\hline & $\begin{array}{l}\text { Áreas geográficas recibidas en el } \\
\text { Instituto }(* * *) \text { p. } 25(364)\end{array}$ & \\
\hline
\end{tabular}

\begin{tabular}{|c|c|}
\hline \multirow{7}{*}{ Área $G^{a}$ Física } & Climatología (92) \\
\hline & Medio Ambiente (34) \\
\hline & Naturaleza (80) \\
\hline & Geomorf y Geología (36) \\
\hline & Fauna y Flora (22) \\
\hline & Geografía Física (41) \\
\hline & Otros (8) \\
\hline \multirow{8}{*}{ Área $G^{a}$ Humana } & Demografía (15) \\
\hline & Población (48) \\
\hline & Sociología (15) \\
\hline & Economía (53) \\
\hline & Urbanismo (21) \\
\hline & Política (30) \\
\hline & Geografía Humana (23) \\
\hline & Otros (35) \\
\hline \multirow{5}{*}{ Área $\mathrm{G}^{\mathrm{a}}$ Regional } & Territorio (20) \\
\hline & Tierra (57) \\
\hline & Paisaje (21) \\
\hline & Geografía Regional (19) \\
\hline & Otros $(20)$ \\
\hline \multirow{2}{*}{ A. Técnicas } & Cartografía (82) \\
\hline & Otros $(8)$ \\
\hline Otras disciplinas & Historia (17) \\
\hline \multirow{2}{*}{ Adjetivos } & Positivos (70) \\
\hline & Negativos (5) \\
\hline
\end{tabular}


Este análisis de contenido, según Bisquerra y Sabariego (2004), nos permite situarnos ante la investigación, para tener una primera impresión y visualización de quienes participan y de su relación con el objeto de estudio, de tal manera que podamos elaborar un marco de actuaciones futuras. Las encuestas cualitativas «son instrumentos que nos sirven para descubrir cuáles son los significados de los constructos utilizados por cada persona, son pruebas proyectivas, en las que nos vierten los significados que las personas encuestadas atribuyen a determinados conceptos» (Goetz y Lecompte, 1988, p. 126). Y así, al estar las preguntas elaboradas de forma abierta la información que se obtiene puede ser «rica en matices». (Bisquerra y Sabariego, 2004, p. 337).

Los instrumentos: se elaboró un cuestionario a través de la herramienta que proporciona Google en el que se incluían las siguientes preguntas: Orden de la elección del Grado o la Licenciatura de Geografía en la matrícula de ingreso a la universidad, ¿Por qué has elegido estudiar Geografía?, ¿Qué tipo de estudios esperabas encontrar?, ¿Qué entiendes que es la Geografía?, define cinco palabras que crees que encierra la Geografía como disciplina universitaria y de conocimiento, ¿cuáles son las ramas de la Geografía que más te interesan?, ¿En cuál de las tres ramas te sientes más cómodo/a? ¿Qué contenidos de la materia de Geografía recuerdas de tú época de instituto, ikastola...? Se ha utilizado un cuestionario elaborado expresamente para esta investigación que ha sido testada con un grupo, antes de ser aplicada a todo el alumnado, ya que, según Goetz y LeCompte (1988, p .135), antes de aplicar una encuesta se debe tener en cuenta si las preguntas tienen sentido para quien va a responder y si suscitan la aparición de los datos buscados.

Participantes: La Investigación ha sido llevada a cabo durante los cursos escolares 201112 y 2012-13 en la Facultad de Letras de la UPV-EHU. Ha participado el alumnado del Grado de Geografía y Ordenación del Territorio y de la Licenciatura de Geografía. Durante el curso 11-12, de los 107 alumnos matriculados 77 han completado, es decir el $72 \%$ del alumnado matriculado. En el curso 2012-13 el cuestionario ha sido completado únicamente por los estudiantes de primer curso donde el porcentaje de respuestas se ha elevado al $88 \%$ respondiendo 38 de los 43 estudiantes como queda reflejado en la Tabla 1. En resumen, de los 150 alumnos y alumnas matriculadas en el momento de la encuesta 117 la han completado, siendo el porcentaje de participación del $78 \%$.

Tabla 1

NÚMERO DE RESPUESTAS OBTENIDAS POR CICLO

\begin{tabular}{lcccc}
\hline \multicolumn{1}{c}{ CURSO } & \multicolumn{2}{c}{ PARTICIPANTES } & \multicolumn{2}{c}{ TOTAL ALUMNOS } \\
& $\mathrm{N}^{\mathrm{o}}$ & $\%$ & $\mathrm{~N}^{\mathrm{O}}$ & $\%$ \\
\hline $1^{\circ}$ curso Grado 12-13 & 38 & $25 \%$ & 43 & $29 \%$ \\
\hline $1^{\circ}$ curso Grado 11-12 & 30 & $20 \%$ & 38 & $25 \%$ \\
\hline $2^{\circ}$ curso Grado 11-12 & 23 & $15 \%$ & 30 & $20 \%$ \\
\hline $3^{\circ}$ curso Licenciatura 11-13 & 9 & $6 \%$ & 14 & $9 \%$ \\
\hline $4^{\circ}$ curso Licenciatura 11-12 & 17 & $11 \%$ & 25 & $17 \%$ \\
\hline Total & $\mathbf{1 1 7}$ & $\mathbf{7 8 \%}$ & $\mathbf{1 5 0}$ & $\mathbf{1 0 0 \%}$ \\
\hline
\end{tabular}

Fuente: elaboración propia. 
Procedimiento (desarrollo de la investigación): Todo el alumnado de Geografía tuvo acceso al cuestionario. La mayoría lo completó en una de las horas de clase en el aula de informática solicitada para tal efecto, a los que no asistieron a clase se les envió el enlace mediante correo electrónico. Una vez analizada la información obtenida se elaboró el correspondiente informe. Las respuestas se han codificado mediante el siguiente código: AL113MP12C1-13 donde $A L=$ alumno; $113=n^{\circ}$ de alumno; $M=$ masculino; $P=$ pregunta $;$ $12=n^{o}$ de pregunta $; C=$ curso $; 1=$ primero y 13 en este caso hace referencia a los estudiantes matriculados en primero durante el curso 12-13.

\section{RESULTADOS}

En este apartado recogemos las razones que llevaron al alumnado a elegir los estudios de Geografía, las ideas previas y las expectativas que tenían con respecto a dichos estudios, qué definición propusieron de la Geografía y qué términos asocian a esta disciplina, así como, cuál es su área geográfica preferida. Para completar esta información se recogen datos acerca de cuáles han sido las materias recibidas en el instituto relacionadas con esta ciencia y qué posición ha ocupado la Geografía a la hora de elegir sus estudios universitarios.

\section{III.1. Razones que Ilevaron a los estudiantes a elegir el Grado de Geografía}

Entre las razones que argumentan para la elección de sus estudios encontramos razones de índole personal, razones profesionales, académicas o relacionadas con su entorno social.

Entre las razones de índole personal (RPS) en la Tabla 2, se recogen las respuestas que hacen referencia a sus aficiones, a su inquietud y disponibilidad por aprender o al interés directo por la Geografía. Dentro de este apartado prima el interés que tienen hacía la propia carrera, ya que la consideran de gran utilidad para comprender el mundo que nos rodea. Si bien, cuando hablan de sus aficiones, éstas van encaminadas a la parte más física de la disciplina, es decir, hacia el interés por la naturaleza y la montaña.

«Porque tengo interés en la montaña y la naturaleza. Y porque también tengo ganas de viajar». AL13MP12C2

Las razones profesionales (RProf) que han llevado al alumnado a elegir estudiar Geografía están relacionadas con una perspectiva laboral optimista o para mejorar el currículo. Consideran que los cambios que incluye el Grado respecto a la Licenciatura pueden suponer una ventaja a la hora de acceder al mercado laboral

«Porque viendo la situación de los estudiantes de otras carreras hoy en día, supuse que al ser carrera nueva y con un poco de suerte, siendo de la primera promoción, no tendría problemas a la hora de entrar en el mundo laboral». AL16FP12C2

«Ya que considero que es una carrera muy completa, mediante la cual se adquieren conocimientos que considero muy importantes. Además, ofrece diferentes sali- 
das profesionales, salidas que no se limitan en solo campo. A su vez, al no haber demasiada gente realizando esta carrera, es posible que resulte más sencillo conseguir un trabajo». AL103FP12C1-13

Las razones académicas (RA), tienen que ver con no ser admitidos en otras carreras por la nota de selectividad, y se antoja como una razón importante para optar por matricularse en Geografía, ya que es una carrera sin nota de acceso. En la propia Facultad esto queda reflejado en el aumento de matrículas que hay respecto al número de prematriculaciones.

«En verdad, no elegí Geografía en la primera opción, sino que lo que a mí me interesaba era Trabajo Social, pero al venir de $2^{a}$ convocatoria, las dos carreras que puse antes de Geografía ya no tenía opción a entrar en ellas». AL35FP12C1-12 «Mi primera opción fue historia, pero por la nota finalmente no pude entrar, y puse Geografía como segunda opción porque me gusta todo lo que tenga que ver con el mundo, las tierras, los mapas...». AL100FP12C1-13

Si analizamos la figura 1 podemos comprobar que únicamente el 58\% del alumnado escogió Geografía como primera opción. Este dato resume las razones académicas que han impulsado al alumnado a matricularse en el Grado o la Licenciatura.

Tabla 2

ORDEN DE ELECCIÓN DEL GRADO O LA LICENCIATURA DE GEOGRAFÍA EN LA MATRÍCULA DE INGRESO A LA UNIVERSIDAD DEL ALUMNADO QUE HA RESPONDIDO A LA ENCUESTA

\begin{tabular}{|c|c|c|c|c|c|c|c|c|}
\hline & \multicolumn{2}{|c|}{$1^{a}$ opción } & \multicolumn{2}{|c|}{$2^{a}$ opción } & \multicolumn{2}{|c|}{$\begin{array}{l}3^{\text {a }} \text { opción o } \\
\text { posteriores }\end{array}$} & \multicolumn{2}{|c|}{$\begin{array}{c}\text { Total } \\
\text { estudiantes } \\
\text { por Curso }\end{array}$} \\
\hline & $\mathbf{N}^{\circ}$ & $\%$ & $\mathbf{N}^{o}$ & $\%$ & $\mathbf{N}^{\mathbf{o}}$ & $\%$ & $\mathbf{N}^{\mathbf{0}}$ & $\%$ \\
\hline $1^{\circ}$ Grado $12-13$ Castellano & 8 & $7 \%$ & 6 & $5 \%$ & 0 & $0 \%$ & 14 & $12 \%$ \\
\hline $1^{\circ}$ Grado $12-13$ euskera & 16 & $14 \%$ & 7 & $6 \%$ & 2 & $2 \%$ & 25 & $21 \%$ \\
\hline $1^{\circ}$ Grado $11-12$ castellano & 4 & $3 \%$ & 5 & $4 \%$ & 1 & $1 \%$ & 10 & $9 \%$ \\
\hline $1^{\circ}$ grado $11-12$ euskera & 11 & $9 \%$ & 5 & $4 \%$ & 4 & $3 \%$ & 20 & $17 \%$ \\
\hline $2^{\circ}$ Grado $11-12$ castellano & 5 & $4 \%$ & 2 & $2 \%$ & 2 & $2 \%$ & 9 & $8 \%$ \\
\hline $2^{\circ}$ Grado $11-12$ euskera & 7 & $6 \%$ & 5 & $4 \%$ & 2 & $2 \%$ & 14 & $12 \%$ \\
\hline $3^{\circ}$ Licenciatura $11-13$ castellano & 3 & $3 \%$ & 2 & $2 \%$ & 0 & $0 \%$ & 5 & $4 \%$ \\
\hline $3^{\circ}$ Licenciatura $11-13$ euskera & 4 & $3 \%$ & 0 & $0 \%$ & 0 & $0 \%$ & 4 & $3 \%$ \\
\hline $4^{\circ}$ Licenciatura $11-12$ castellano & 5 & $4 \%$ & 2 & $2 \%$ & 0 & $0 \%$ & 7 & $6 \%$ \\
\hline $4^{\circ}$ Licenciatura $11-12$ euskera & 5 & $4 \%$ & 3 & $3 \%$ & 1 & $1 \%$ & 9 & $8 \%$ \\
\hline Total estudiantes por opción & 68 & $\mathbf{5 8 \%}$ & 37 & $32 \%$ & 12 & $10 \%$ & 117 & $100 \%$ \\
\hline
\end{tabular}

Fuente: elaboración propia. 
Pero también las experiencias escolares previas son importantes y pueden resultar determinantes, tanto relacionadas con la materia como con el profesorado.

«El motivo por el que elegí estudiar esta carrera es porque disfrutaba mucho en las clases de Ciencias Sociales de la ESO y en Geografía de bachillerato. Lo que más me gustaba era el trabajo con mapas, ya que me parecían muy representativos y no me costaba mucho memorizarlos. Asimismo, me gustaba escoger un territorio y analizar relieve, ríos, capitales y ciudades más importantes, población...» AL1MP12C3 UNIVERSIDAD DEL ALUMNADO QUE HA RESPONDIDO A LA ENCUESTA

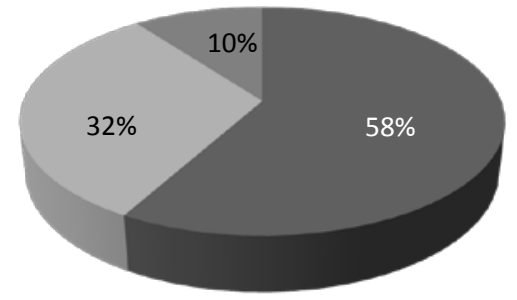

$$
\begin{aligned}
& \text { Como } 1^{\mathrm{a}} \text { opción } \\
& \text { Como } 2^{\mathrm{a}} \text { opción } \\
& \text { Como } 3^{\mathrm{a}} \text { opción o } \\
& \text { posteriores }
\end{aligned}
$$

Fuente: elaboración propia.

Una última razón académica es que el propio contenido de la carrera les resulta atractivo. Los estudiantes conocen o se han informado acerca de la materia que se va a impartir y es eso lo que les anima a matricularse.

«Me parece interesante el conocimiento que puedes adquirir con esta carrera para entender más lo que te rodea, tanto lo natural como lo que el hombre ha construido». AL96FP12C1-13

«No tenía ninguna otra en mente, vi el temario y sus salidas y me gustó». AL43FP12C2

Las razones relacionadas con su entorno social (ES), tienen que ver con la familia o los amigos pueden ser o son un pilar importante a la hora de elegir unos u otros estudios universitarios. En algunos casos, los amigos y en otros, la familia, tienen mucha influencia a la hora de elegir los estudios Universitarios.

«Mi aita es geógrafo, él es quien me ha inculcado el interés hacia la Geografía». AL10MP12C4

«Porque desde pequeño me ha gustado mucho y ha sido una afición que he compartido con mi padre». AL94MP12C1-13

En la Figura 3 y Tabla 3 podemos comprobar cómo se han distribuido las 117 respuestas obtenidas en esta pregunta. 


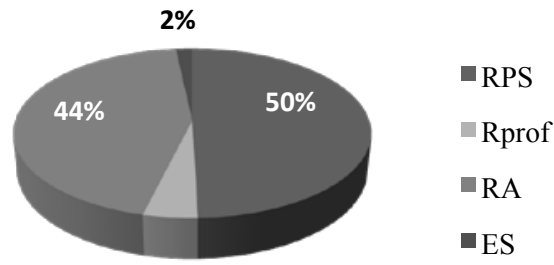

Fuente: elaboración propia.

Tabla 3

$N^{\circ}$ DE RESPUESTAS POR CATEGORÍA

\begin{tabular}{lcc}
\hline & $\mathrm{N}^{\mathrm{a}}$ & $\%$ \\
\hline RPS & 58 & $50 \%$ \\
\hline Rprof & 5 & $4 \%$ \\
\hline RA & 52 & $44 \%$ \\
\hline ES & 2 & $2 \%$ \\
\hline Total & 117 & $100 \%$ \\
\hline
\end{tabular}

Fuente: elaboración propia.

\section{III.2. Estudios geográficos, relacionado con ideas previas y expectativas}

Al preguntar qué tipo de estudios esperaban encontrar al llegar a la Facultad, fueron numerosas las respuestas que expresaron no haber encontrado lo que esperaban. Pero en otros casos, más que hablar de lo que esperaban, mencionaron lo que no esperaban encontrar y han tenido que estudiar. Esto último, como ya veremos más adelante, está estrechamente ligado al Grado de Geografía y Ordenación del Territorio, ya que dentro del grupo de asignaturas básicas se comparten materias con los Grados de Historia e Historia del Arte y se imparten asignaturas de Filosofía y Expresión Oral y Escrita, materias estas últimas que no se impartían en la Licenciatura.

Entre lo que esperaban, un $25 \%$ del alumnado ha respondido haberse encontrado en la carrera unos estudios acordes a sus expectativas, lo cual demuestra que el trabajo de información previo a las matriculaciones o la información a la que tiene acceso el futuro alumnado universitario, ayudan a dar a conocer la materia a recibir.

«Bastante semejantes a los que me he encontrado en la realidad. Tenía conocimiento de que se trata de una carrera que alberga temas muy variados». AL10MP13C4 «Básicamente los que he encontrado. Geografía Física, Geografía Urbana, Climatología, Geografía Humana». AL71MP13C4

Hay también quién no esperaba nada en especial, mientras algunas personas tienen muy claro que es lo que se van a encontrar, en otros casos vienen a probar y experimentar sin saber qué es lo que van a estudiar (4\% de las respuestas). 
«No esperaba encontrar nada en especial. He venido con la mente muy abierta, con ganas de dejarme convencer, de escuchar cualquier cosa que sea nuevo en mis conocimientos,...» ALO2FP13C3

Se han encontrado todo tipo de respuestas, también algunas que no se esperaban, tal y como apuntaban Goetz y LeCompte (1988), y así hay quienes califican los estudios como atractivos, elaborados, interesantes, prácticos, fáciles... (11\% de las respuestas).

«Elaborados, de materia interesante y práctica». AL66MP13C1-12

Determinadas respuestas apuntan a que esperaban una continuación del bachillerato, y destacan que la materia recibida en el instituto es importante para conocer las diferentes ciencias existentes. En el caso de la Geografía, en función del tipo de Bachillerato escogido se imparte como asignatura propia o comparte protagonismo con la Historia. Aunque hay estudiantes que no han cursado la asignatura, sí que para otros, ha supuesto un punto de referencia a la hora de presuponer que es lo que se iban a encontrar en la Facultad. (2\% de las respuestas).

«Esperaba encontrar algo parecido a lo dado en geografía en Bachillerato, pero es completamente diferente. También me imaginaba que sería algo más fácil, además de dar asignaturas referidas con Geografía...». AL35P13C1-12

«Estudios que profundizasen lo estudiado en la ESO y Bachiller, y con miras más globales». AL51MP13C4

Hay también respuestas relacionadas con otras áreas, dentro del grado de Geografía y Ordenación del Territorio se comparten asignaturas con otras titulaciones como Historia, Arte o Filosofía, teniendo entre primer y segundo curso una carga de créditos importante de asignaturas «no geográficas». En la Licenciatura, sin embrago, el porcentaje de asignaturas no geográficas es mucho menor. Este hecho queda patente en las respuestas obtenidas, ya que de la totalidad de respuestas de este apartado un $21 \%$ del total hacen referencia al exceso de asignaturas no relacionadas directamente con la Ciencia Geográfica, tales como Historia, Filosofía, Expresión oral y Escrita o Economía, y todas corresponden a estudiantes de primer o segundo curso Consideran que la carga de créditos que suponen estas asignaturas sobre el total del Grado puede mermar la cualificación como geógrafos de cara a su futura inserción laboral.

«Estudios más centrados en la Geografía sabía, antes de matricularme, de la existencia de asignaturas no relacionadas con esta rama, pero he de admitir que me he llevado una gran desilusión, pues pienso, que aunque interesantes, muchas de las asignaturas no son válidas o son poco productivas para un futuro profesional como geógrafo». AL37MP13C1-12

«Esperaba más asignaturas relacionadas con la Geografía, ya que en primer y segundo curso hemos tenido muchas asignaturas de Historia y Arte, y eso me desanimó un poco». AL15FP13C2 
Entre las respuestas relacionadas con las áreas de Geografía, más de un tercio del alumnado, (el 38\%), ha respondido que esperaba encontrar en el Grado o la Licenciatura asignaturas relacionadas con las diferentes áreas geográficas. Hay que recordar que, a las tradicionales áreas de Geografía Física y Humana, hay que añadir el área de Geografía Regional y el área de las asignaturas técnicas (cartografía, SIG,...). De los cuatro apartados, el de Geografía Física, es el que aparece con más frecuencia entre las respuestas proporcionadas.

«Me esperaba encontrar unos estudios de carácter mucho más descriptivo, más relacionados quizás con la denominada geografía regional. Por ejemplo, en primer curso lamentaba que sólo existiera geografía de Europa, de España y de Euskal Herria como regional en toda la carrera. Me habría gustado en ese momento la existencia de geografías de otros continentes. AL01MP13C3

«No tenía una idea fija pero sobre todo esperaba dar asignaturas relacionadas con la población humana». AL46FP13C2

«Los diferentes estudios que hay dentro de la Geografía Física, a decir verdad, me los esperaba aprender (geomorfología, clima...) lo que más me ha sorprendido es, los diferentes estudios que estamos haciendo dentro de la Geografía Humana. Yo, desde un principio no me esperaba que tuviéramos una asignatura sobre ciudades, o tampoco sobre el ámbito rural». AL03MP13C3

En el cuestionario se les pregunta por los conceptos geográficos relacionados con la Geografía que han estudiado en su etapa de Instituto o Ikastola. Es una pregunta abierta, donde pueden mencionar todas las materias que recuerden. Pero ha destacado un tema que ha tenido un peso importante en los estudios tradicionales de Geografía, que es el «relieve» que junto a las «capitales» son términos estos asociados a la Geografía clásica y no tanto a la Geografía moderna, o más científica, pero que siguen teniendo un peso muy importante en la enseñanza secundaria. Los 117 participantes han recordado de 2 a 4 materias cada uno, siendo el total de respuestas de esta pregunta 364 materias recibidas en su época de instituto, que hemos agrupado en diferentes categorías y que se muestran en la figura 3, donde a las ya mencionadas «relieve» y «capitales», hay que añadir la Climatología, la Cartografía, la $G^{a}$ Física, la Geografía de la Población, la Geografía Política o los temas relacionados con la Economía como materias más importantes.

\section{III.3 ¿Qué entienden por Geografía hoy en día?}

Los participantes son alumnos de $1^{\circ}, 2^{\circ}, 3^{\circ}$ y $4^{\circ}$ por lo que el bagaje geográfico que cada uno posee es muy diferente para dar una definición de la Geografía lo más completa posible, aun así, han sido muy enriquecedoras las respuestas obtenidas. En unos casos, se han limitado a definir la ciencia geográfica mediante adjetivos y en otros, han profundizado en el objeto de estudio de la Geografía. A esta pregunta han respondido los 117 participantes.

La Geografía como arma política, aunque solo ha habido una respuesta en esta categoría me parece interesante destacarla, ya que hoy en día la Geografía ocupa un lugar muy importante en la gestión del territorio, y dicha gestión está controlada por los políticos que están en el poder. 


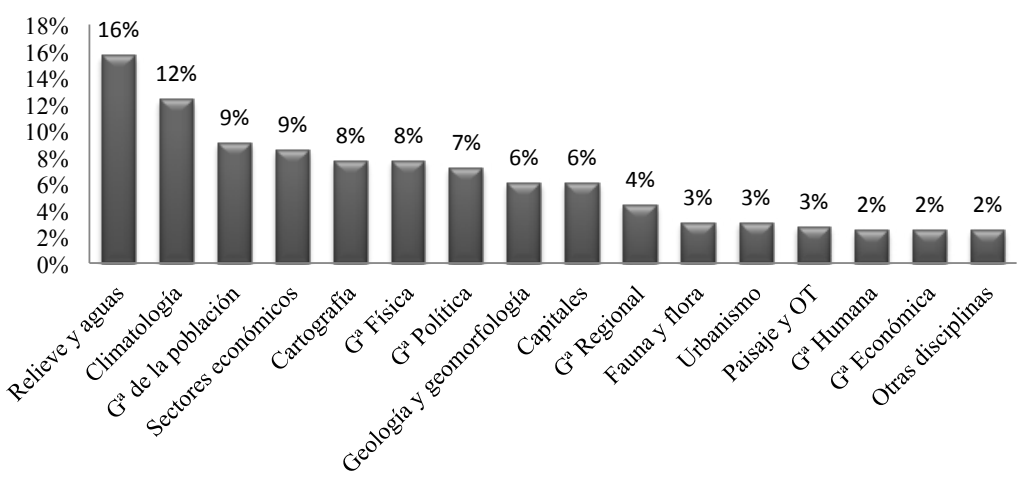

Fuente: elaboración propia.

«Un arma para la guerra y la lucha política». AL22MP19C2

La búsqueda de respuestas de la realidad, es otra de las que ha recibido una única respuesta, es importante, porque no supone una mera descripción, por eso hay que destacar que alguien haya mencionado la búsqueda del «por qué» de la realidad.

«Disciplina atractiva. Qué busca, el porqué de la realidad y no la elaboración vacía de documentos». AL07FP19C4

Entienden que supone un Estudio de la Tierra/Planeta, en concreto, el 76\% de las respuestas obtenidas por los participantes se enmarcan dentro de esta categoría, por lo que queda claro que para los participantes el objeto de estudio de la Geografía, es la Tierra, el Planeta o la superficie Terrestre. Dentro de esta categoría encontramos las referencias al Planeta, al estudio de su evolución, o de su parte física, la interrelación entre los elementos, de la interpretación o del estudio integral del territorio.

Existen 23 respuestas referidas al Planeta, Si bien hemos llamado a esta categoría Planeta, hemos incluido los conceptos de «superficie terrestre» y «tierra» ya que han sido empleados bajo el mismo concepto o categoría. Consideran que la Geografía ha de estudiar, comprender o analizar la superficie terrestre.

«La ciencia que estudia la tierra con todo lo que ello implica». AL76FP19C2

«La ciencia que examina y valora la superficie terrestre». AL97MP19C1-13

Tres respuestas referidas al estudio de la evolución del planeta, a pesar de ser estudiantes de geografía los participantes, también han relacionado esta disciplina con la evolución, tarea más propia de geólogos que de geógrafos. Aunque si bien es cierto que la Geografía necesita conocer el origen o la evolución de los procesos, se suele limitar a épocas más recientes. 
«Una manera de conocer dónde estuvimos, dónde estamos y dónde estaremos. Por qué se ha creado esta montaña y este rio y cuáles son sus materiales, su flora su fauna y todo lo que la rodea. Entender el clima y todas sus variantes.» AL31MP19C1-12

Hay dos respuestas referidas a la parte física del Planeta. Como ya hemos visto anteriormente, la Geografía se compone de tres ramas principales, pero es frecuente relacionarla solo con una de ellas, como ocurre en esta subcategoría, donde la parte física predomina frente a la humana o regional.

«Disciplina sobre el estudio del territorio físico: situación, formación, relieve terrestre, litoral etc. Creación y actualización, actualmente los satélites precisan, la cartografía, ubicación».AL33FP19C1-12

Existen 16 respuestas relacionadas con esta subcategoría, interrelaciones de los elementos del Territorio, donde podemos encontrar las definiciones de Geografía que coinciden casi o en su totalidad con la definición que atribuyen los teóricos a la disciplina, ya que la definen como la ciencia que estudia -y no solo describe- las variaciones y distribuciones de los fenómenos de la superficie terrestre -abióticos, bióticos y culturales- y sus relaciones. En todas las respuestas se incluyen las palabras «relaciones» $\mathrm{o}$ «interrelaciones» entre los diferentes componentes de la superficie terrestre.

«Es la ciencia que estudia la superficie terrestre, la distribución espacial de los elementos que en ella se encuentran, así como las interrelaciones de dichos elementos, siempre desde un enfoque espacial y global» AL71MP19C4

«Ciencia que estudia el medio físico y humano y sus interrelaciones». AL23MP19C2

«La geografía es la ciencia que estudia la tierra y las relaciones que se dan en ella tanto físicamente como socialmente y entre estas dos». AL75FP19C4

Las 5 respuestas referidas a la Interpretación del Territorio aquí obtenidas hablan de entender o interpretar el espacio en que vivimos.

«La Geografía es básica para entender qué tenemos alrededor nuestro. Estamos conviviendo con el clima, los paisajes...» AL32P19

Existen 36 respuestas referidas al Estudio integral del Territorio. Entorno al $31 \%$ de las respuestas de la pregunta 19 quedan enmarcadas dentro de esta subcategoría, dejando patente la importancia que tiene la Geografía para una visión global del territorio según el alumnado. Consideran que la labor de la ciencia Geográfica es estudiar al ser humano (Economía, Historia, Población...), pero también el medio natural (Clima, Geomorfología, Edafología, Hidrología...). Entre las respuestas aparecen las palabras «integral», «general»o «todo» que resumen muy bien el quehacer geográfico. Queda, pues, superada por parte del alumnado esa dicotomía histórica de la Geografía, donde muchos autores hablaban de dos ciencias diferentes e independientes entre sí, es decir, que la Geografía Física y la Humana no tenían que ir de la mano necesariamente. 
«Una ciencia que tiene como propósito mostrar al receptor, los elementos físicos o químicos de la tierra, tanto como, los sistemas políticos, económicos y sociales proyectados a través del ser humano». AL74MP19C1-12

«La visión integral de todo lo que nos rodea, pero sobre todo, un medio para entender el mundo actual, pero esto último, la actualidad es algo que brilla por su ausencia, no preocupa al docente». AL09MP19C4

«Entiendo que es la ciencia que estudia muy diversos fenómenos que ocurren sobre la corteza terrestre (excepto las ciencias que también estudian el interior del planeta), desde físicos (geomorfología, edafología, hidrología, climatología) hasta sociales (geografía humana, geografías regionales, historia económica mundial...)». AL19MP19C2

«Creo que la geografía es conocer y entender el mundo en todos los aspectos». AL101MP19C1-13

Existen otros conceptos que relacionan la Geografía con la definición de CIENCIA. En esta categoría, hemos incluido las respuestas que más que profundizar en el objeto de estudio de la Geografía, hacen referencia a características de la ciencia geográfica en general (el 26\% de las respuestas se incluyen en esta categoría).

La Geografía como Ciencia Social recibe cinco respuestas. Antiguamente la Geografía se estudiaba dentro de la materia «Sociales» con lo que es fácil ubicarla dentro de esta categoría, si bien, hemos comprobado que es una ciencia que se dedica al estudio de lo social, pero también del medio natural.

«La Geografía es la ciencia social que estudia la relación entre los sistemas naturales y humanos, buscando soluciones a los problemas que surgen desde esta relación. Es ciencia porque genera teorías generales, aplicables a casos particulares; y es social porque, a pesar de que estudie los sistemas naturales, debe enfocarse en el ser humano y en el uso que éste hace de su espacio». AL28MP19C3

Dos respuestas hablan de Ciencia indefinida. La respuesta recogida a continuación resume a la perfección una de las mayores inquietudes de los geógrafos, que no es otra que la de buscar una definición que resuma el amplio abanico de estudio de la Geografía. Como hemos podido ver en la teoría desde sus comienzos, la Geografía ha seguido diferentes tendencias sin haber encontrado muchas veces su lugar dentro del mundo científico.

«Es un concepto que ni siquiera los geógrafos más prestigiosos han sabido definir, pero sé que es lo que me gusta y que acerté al matricularme en esta licenciatura». AL5M1P19C4

Once personas encuestadas la definen como una Ciencia multidisciplinar. Otro de los problemas de la Geografía es que no tiene bien delimitado hasta donde abarca su conocimiento, es decir, dónde acaba el quehacer geográfico y dónde empiezan otras disciplinas. Por poner un ejemplo, la Geografía Física estudia la vegetación y su distribución, pero en muchas ocasiones, se encuentra con los biólogos en este tipo de estudios. Y esto lo podemos 
hacer extensible al resto de especializaciones geográficas. Por eso, en las respuestas encontradas en esta subcategoría hacen mención al amplio abanico de estudio de la Geografía y a su relación estrecha con otras ciencias.

«Una ciencia pero que junta cosas de muchas ciencias». AL56MP19C1-12

«Una ciencia que abarca muchos conocimientos de diferentes ciencias, como por ejemplo, historia, geología, medio ambiente, sociología...» AL18MP19C2

«Es una ciencia que estudia el planeta y que establece una relación con algunas otras ciencias como la climatología o la estadística.» AL14MP19C2

Hay quién la define como una Ciencia necesaria (2 resp.). Tras las diferentes reformas educativas llevadas a cabo en las últimas décadas, la Geografía ha pasado a ser una asignatura obligatoria solo en determinadas ramas de bachiller, en otros casos, su estudio se produce dentro de otras asignaturas como pueden ser conocimiento del medio, o junto a Historia... Si bien, dentro de la Universidad tiene un espacio propio, hasta hace dos cursos como Licenciatura y ahora como Grado.

«Es una asignatura que ha tenido que expandirse en todo el mundo. Que es necesaria en nuestro mundo. No es solo el conocimiento de un rio de España o del mundo, de montañas y otros fenómenos, sino una ciencia necesaria.» AL21FP19C2

Se define también como Ciencia analítica (4 resp.), por su capacidad analítica para detectar problemas, determinar ubicaciones...

«Una ciencia donde analiza la tierra». AL53MP19C1-12

«Una ciencia que se encarga de analizar los factores físicos de la tierra, incluyendo los aspectos demográficos.» AL103FP19C1-13

A pesar de realizar la encuesta al alumnado de Geografía algún estudiante sigue viendo esta ciencia como una ciencia meramente descriptiva, (3 resp.), lo cual, puede ser el legado de unos estudios anteriores descriptivos o el asociar la Geografía a su imagen más tradicional, pero ya obsoleta.

«Una ciencia que describe el paisaje y el territorio, desde un punto de vista físico y humano, atendiendo a factores de las ciencias naturales y sociales.» AL27MP19C2

Las últimas tendencias geográficas apuntan a una disciplina de actualidad y necesaria en la Planificación y Gestión del Territorio, por lo que se está convirtiendo en una herramienta imprescindible para la resolución de conflictos en la organización territorial (1 resp.).

«Es una ciencia que investiga para conocer el mundo y detectar y solucionar sus problemas». AL54MP19C1-12 


\section{III.4. Definición de la Geografía por conceptos}

En esta pregunta del cuestionario se ha pedido a cada participante que proponga cinco términos que considere que describen esta ciencia. Nueve estudiantes no han propuesto ningún término, por lo que del total de 540 respuestas que podíamos obtener de los 108 estudiantes restantes, estos han sugerido 516. Las personas encuestadas utilizan numerosos términos relacionados con las áreas geográficas (ver Figura 5), pero también aparecen adjetivos negativos como rara, poco práctica, superficial o adjetivos positivos como bonita, enriquecedora, ilusionante o intrigante entre otros. También son numerosos los conceptos relacionados con la geografía, que si bien no se pueden enmarcar dentro de las áreas geográficas, si forman parte del día a día de mundo geográfico, entre estos términos cabe destacar «ordenación o gestión del Territorio», «espacio», «análisis», «relaciones o interrelaciones», «investigación», «diversidad», «descripción».

En la Figura 5, además del número de menciones que ha tenido cada término se han añadido los datos de la Figura 4, ya que la comparación entre ambos resultados es interesante de cara a resaltar la importancia de la materia recibida en el Instituto y la definición de Geografía que proporcionan hoy en día.

Si analizamos estas comparaciones por áreas, podemos ver como en el área de Geografía Física la Climatología, el medio ambiente o la Fauna y Flora son términos que aparecen en las dos preguntas en números bastante similares. En el área de Geografía Humana, la Población es mencionada casi por igual en ambos casos, mientras que la economía, sí que es una materia que recuerdan de su época de Instituto, pero que no la relacionan tanto con la Geografía hoy en día, a pesar de ser una asignatura que todos los estudiantes que han participado en la encuesta han cursado, ya que se imparte durante el primer cuatrimestre de primero.

Figura 5

TÉRMINOS PROPUESTOS PARA DEFINIR LA GEOGRAFÍA Y MATERIA RECIBIDA EN EL INSTITUTO RELACIONADA CON DICHA CIENCIA

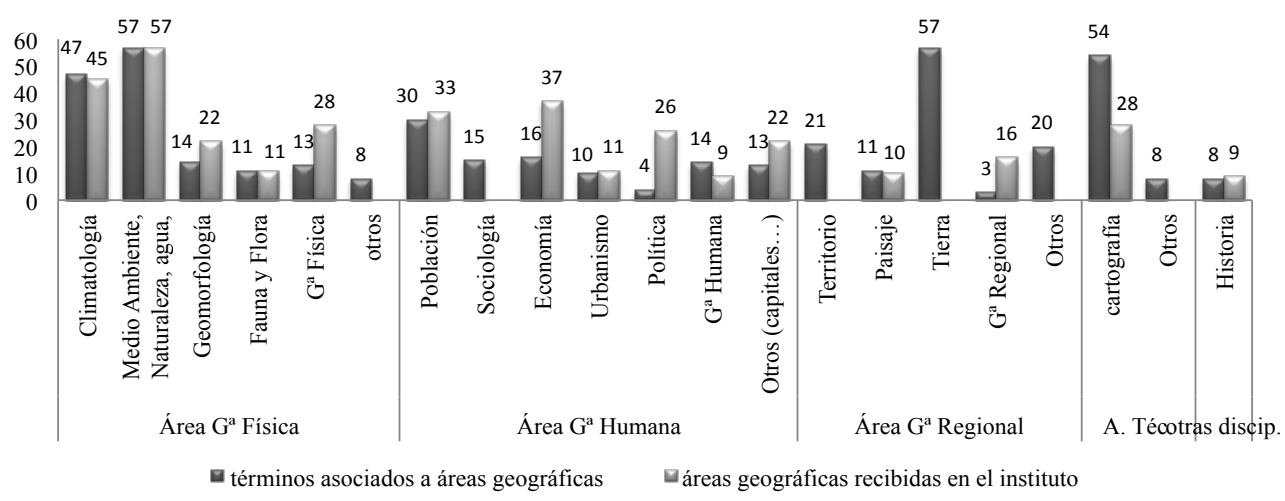

Fuente: elaboración propia.

En el área de Geografía Regional destacan sobre manera los términos relacionados con la Tierra (Superficie Terrestre, Mundo...), mientras que el Paisaje o el Territorio y su gestión 
son términos que han relacionado con la Geografía a partir de su época universitaria. Cabe resaltar la Cartografía, quehacer por excelencia desde sus orígenes de los geógrafos y geógrafas cómo ya señalaban Plans (1984) o Menéndez y Molinero (1991) entre otros.

Por último, mencionar la Historia, ya que a pesar de ser estudiantes del Grado o la Licenciatura, siguen asociando los estudios geográficos con esta disciplina Este hecho puede estar relacionado con que la geografía se ha incluido en el currículo escolar junto a la Historia y que en el propio grado o la Licenciatura sean varias las asignaturas de esta rama científica, las que los estudiantes tienen que cursar.

\section{DISCUSIÓN DE LOS RESULTADOS Y CONCLUSIONES}

La Geografía es una opción minoritaria entre los estudiantes que acceden a la Universidad, por eso, es importante conocer el perfil del alumnado para poder desarrollar estrategias de captación adecuadas. En muchos casos, los alumnos de Bachiller no saben que es lo que se estudia en este Grado o Licenciatura, su conocimiento geográfico se limita al libro de texto empleado en clase.

Mediante la encuesta, hemos podido constatar qué en unos casos son razones personales relacionadas con el gusto por la naturaleza, las que les hacen decantarse por el estudio de esta disciplina, esto se puede deber al cambio dado en la sociedad respecto a una mayor sensibilidad con la naturaleza, mayor accesibilidad y conocimiento de la misma. En otras ocasiones es la experiencia positiva vivida en el Instituto respecto a la materia.

Pero dentro de las razones académicas, cabe destacar que la baja nota obtenida en selectividad les ha impedido acceder a la opción deseada matriculándose en Geografía (el 42\% de los alumnos acceden no siendo la Geografía su primera opción), por lo que los resultados no son demasiado buenos. No hay que olvidar que la tasa de abandono puede estar relacionada con esta cuestión, si lo que se está estudiando no gusta es muy probable que el alumnado se desmotive y no quiera continuar.

En el estudio de Esparcia y Sánchez (2012) ya se hacía referencia a este hecho, indicando que la Geografía era refugio de segundas, terceras y hasta cuartas opciones para los estudiantes.

La segunda conclusión que se ha obtenido es que a pesar de la difusión de información, tan solo el $25 \%$ ha encontrado en la carrera lo que pensaba o esperaba encontrar. El otro $38 \%$ de los participantes esperaban encontrar en el Grado o en la Licenciatura asignaturas relacionadas con las áreas geográficas, sin concretar si realmente conocían la materia que iba a ser impartida. Aunque el dato más preocupante es que el $21 \%$ restante considera que hay una excesiva carga de asignaturas no geográficas que pueden desmotivar o mermar su preparación de cara a una futuro laboral. Los datos obtenidos en esta conclusión coinciden con el estudio realizado por Garrido, Galindo, García, y López (2011) anteriormente mencionado, donde queda patente que el alumnado que accede a los grados de Geografía no sabe con exactitud lo que se va a encontrar. Todo ello, a pesar de la difusión de información que entre los preuniversitarios, realiza en este caso la UPV-EHU en general y la Facultad de Letras mediante su Vicedecanato de Alumnado y el Departamento de Geografía, Prehistoria y Arqueología en particular. Esta promoción del Grado de Geografía y Ordenación del Territorio se lleva a cabo a través de Jornada de Puertas Abiertas, Ferias, Charlas, etc. 
En cuanto a la búsqueda de una definición de la Geografía, ha quedado claro que, para este grupo de estudiantes, es una ciencia que tiene como principal objeto el estudio integral del planeta o la superficie terrestre, y las relaciones que existentes entre el medio humano y físico. Por lo que la consideran una ciencia multidisciplinar. Está pregunta se puede analizar mas exhaustivamente de cara a una futura investigación ya que muy probablemente al analizar las respuestas por curso encontremos diferencias.

En cambio, cuando mediante la encuesta se les pregunta por términos que nos puedan ayudar a definir la Geografía, los más utilizados son Climatología, Territorio o Cartografía. Términos muy ligados a la Geografía tradicional y que dejan patente que las ideas previas que el alumnado posee están muy arraigadas. Estás ideas previas se han construido no sólo gracias al contenido de las materias recibidas en la época anterior a la Universidad. Si no también, como define Rayas (2004), gracias a la interacción con el medio y a las experiencias de la vida cotidiana, pudiendo ser un obstáculo en la comprensión del conocimiento.

En la pregunta que busca conocer cuáles han sido esas materias, la respuesta más repetida ha sido el estudio del relieve y la naturaleza, si bien en esta pregunta también aparecen otras disciplinas de la Geografía como la Economía, o la Política, siguen estando detrás de las tradicional Climatología, aunque por delante de la Cartografía y el estudio de las capitales.

Por lo tanto, relacionan la materia recibida en el Instituto con el Grado o la Licenciatura en Geografía, limitándolo a un estudio más tradicional del que realmente se pretende impartir.

En el Grado, a diferencia de la Licenciatura, la Ordenación y Gestión del Territorio tiene un peso importante, por lo que las futuras generaciones de geógrafos y geógrafas tendrán el estudio integral del territorio, como quehacer profesional superando esa terminología clásica que asocia la Geografía con los mapas, el relieve o el clima.

Esta primera toma de contacto con la investigación cualitativa nos ha servido para certificar algunas conductas que se repiten año a año en los nuevos matriculados, por lo que puede ser el origen de una investigación más amplia.

Así pues, se puede concluir diciendo que los alumnos respecto al Grado o la Licenciatura de Geografía:

- Eligen sus estudios en base a dos razones principales, por un lado tener un verdadero interés por la materia y por otro, haber obtenido una baja nota en selectividad.

- Cuando al alumnado se le pide definir la Geografía la expresión más utilizada es «estudio de las relaciones del Territorio»

- Cuando se le pide que defina la Geografía mediante términos los más empleados son: Climatología, Cartografía y Territorio

- La materia que mejor recuerdan de su época de Instituto relacionada con la Geografía es: Naturaleza-relieve, Climatología, Economía, Política y Territorio.

- Los estudiantes de Grado, a diferencia de los de Licenciatura, muestran cierto descontento con las asignaturas que no pertenecen al área de Geografía propiamente dicha (Arte, Filosofía...). 


\section{BIBLIOGRAFÍA}

AISENSON, D., MONEDERO, F., BATLE, S., LEGASPI, L., AISENSON, G., VIDONDO, M., NICOTRA, D., VALENZUELA, V., DAVISON, S. y ALONSO, D. (2004): Representaciones de estudiantes y graduados recientes sobre la carrera y la profesión del psicólogo. Facultad de Psicología-UAB. Secretaría de Investigaciones y la profesión del psicólogo. XII Anuario de Investigaciones.

ANECA (2004): Libro Blanco para el diseño del Título de Grado de Geografía y Ordenación del Territorio. Madrid. Aneca

BALDUZZI, M.M. y CORRADO, R.E. (2010): Representaciones sociales e ideología en la construcción de la identidad profesional de estudiantes universitarios avanzados. Revista Intercontinental de Psicología y Educación, 12 (2), 65-83.

BISQUERRA ALZINA, R. y SABARIEGO PUIG, M. (2004): Fundamentos metodológicos de la investigación educativa. In R. Bisquerra (Coord.) Metodología de la investigación educativa (1st ed., pp. 20-49). Madrid: La Muralla.

CALLEJO, J. (2001): El grupo de discusión: introducción a una práctica de investigación. Barcelona: Ariel Practicum.

CAMARERO RIOJA, LA. y OLIVA SERRANO, J. (2002): Urban sprawl, rural turnaround and the changing shape of utopia. Explaining counter-Urbanization: Historical approaches to Urban-Rural migration. XIIIth Word Congress of the International Economic History, Buenos Aires.

CAMARERO RIOJA, L.A. y OLIVA SERRANO, J. (2008): Exploring the social face of urban mobility: daily mobility as part of the social structure in Spain. International Journal of Urban and Regional Research, 32 (2), 344-362.

CAPEL, H. (1981): Filosofía y ciencia en la Geografía contemporánea. Barcelona: Barca nova.

CAPEL, H. (1998): Una Geografía para el siglo XXI. Scripta Nova Rev. Electrónica de Geografía y Ciencias Sociales, 19. En http://www.ub.edu/geocrit/sn-19.htm Última revisión 2013/06/04.

COLEGIO OFICIAL DE GEÓGRAFOS (2008): Perfiles profesionales de los geógrafos en España. Disponible en: http://www.geografos.org/images/stories/interes/perfiles/ Perfiles_profesionales.pdf última revisión 21/06/13

CUBERO, R. (1994): Concepciones alternativas, preconceptos, errores conceptuales.... ¿Distinta terminología y un mismo significado? Investigación en la escuela 23, 33 -41.

DEITINGER, P., NARDELLA, C., BENTIVENGA, R., GHELLI, M., RONCHETTI, M. y BONAFEDE, M. (2009): Mundo laboral y jóvenes en Italia: estudio cualitativo sobre imágenes y representaciones. Revista Psicologías, Vol 1. Disponible en http://psicologias.uprrp.edu/articulos/Le_appresentazioni.pdf Última revisión 2013/10/25.

DIBARBOURE, M. (2007): Pensando en la enseñanza y sus dificultades. Quehacer educativo, 86, 140-145.

ESPARCIA PÉREZ, J. y SÁNCHEZ AGUILERA, D. (2012): De la teoría a la práctica. El proceso de diseño e implantación de los grados de geografía en las universidades españolas. Boletín de la Asociación de Geógrafos Españoles, 58, 405-428.

ESTÉBANEZ, J. (1982): Tendencias y problemática actual de la Geografía. Madrid: Cincel. 
FERNÁNDEZ CASO, M.V. y GUREVICH, R. (2010): La imagen pública de la Geografía. Una indagación desde las visiones de profesores y padres de alumnos secundarios. Revista bibliográfica de Geografía y Ciencias Sociales, 859 (XV). En http://www. ub.edu/geocrit/b3w-859.htm Última revisión 2013/11/16.

GARCÍA SÁNCHEZ, M.D. (2008): Expectativas de los estudiantes ante la asignatura de historia de la psicología. Enseñanza e investigación en psicología 13 (1), 15-26.

GARCÍA GARRIDO, J.L. (1991): Fundamentos de la educación comparada. Madrid: Dykinson.

GARCÍA GARRIDO, J.L. (1997): Educación comparada en una sociedad global. Revista española de educación comparada, 3, 61-81.

GARRIDO CUMBRERA, M., GALINDO PÉREZ DE AZPILLAGA, L., GARCÍA MARTÍN, M. y LÓPEZ LARA, E. (2011): La imagen de la geografía en la titulación de geografía y gestión del territorio de la universidad de Sevilla: Situación actual (2008-2011) y propuestas para su mejora. En J.J. Delgado, M.L. Lázaro, y M.J. Marrón (Eds). Aportaciones de la geografía en el aprendizaje a lo largo de la vida (pp. 72-82). Congreso Ibérico de Didáctica de la Geografía. Málaga: Universidad de Málaga.

GOETZ, J.P., y LE COMPTE, M. D. (1988): Etnografía y diseño cualitativo en investigación educativa. Madrid: Morata.

GÓMEZ MENDOZA, J. et al. (1998): El pensamiento geográfico: estudio interpretativo y antología de textos Madrid: Alianza.

GUTIERREZ ALBERONI, J.D. (1998): La teoría de las representaciones sociales y sus implicaciones metodológicas en el ámbito psicosocial. Psiquiatría Pública, 10 (4), 211-219.

HALFACREE, K. (1993): Locality and social representation: space discourse and alternative definitions of the rural. Journal of Rural Studies, 9, 23-37.

HALFACREE, K. (1995): Talking about rurality: social representations of the rural as expressed by residents of six English parishes. Journal of Rural Studies, 11, 1-20.

HALFACREE, K., y BOYLE, P. (1998): Migration, rurality and the post-productive countryside. En P. Boyle y K. Halfacree (Eds.) Migration into rural areas. Theories and Issues, (pp. 1-20). Chichester: Wiley.

HOLT JENSEN, A. (1992): Geografía, Historia y Conceptos. Barcelona: Vicens-Vives.

HUMBOLDT, V.A. (2011): Cosmos. Ensayo de una descripción física del mundo. Madrid: Catarata.

JHONSTON, R.J. y CLAVAL, P. (1986): La Geografía actual: geógrafos y tendencias. Barcelona: Ariel.

JODELET, D. (1986): La representación social: fenómenos, concepto y teoría. En S. Moscovici (coord.). Psicología Social II. Pensamiento y vida social. Psicología social y problemas sociales. Barcelona: Paidós.

KRUEGER, R.A. (1991): El grupo de discusión. Guía práctica para la investigación aplicada. Madrid: Pirámide.

LARSEN, S. C., FOULKES, M., SORENSON, C.J. y THOMPSON, A. (2011): Environmental learning and the social construction of an exurban landscape in Fremont County. Geoforum, 42 (1), 83-93.

LIBRO BLANCO (2005): Título en Geografía y Ordenación del Territorio. Agencia Nacional de Evaluación de la Calidad y Acreditación. Junio 
LIMON, M.R. y CRESPO, J.A. (2002): Grupos de debate para mayores. Guía práctica para animadores. Madrid: Narcea

MARRÓN GAITE, M.J. (2008): Una experiencia de investigación participante con estudiantes de magisterio acerca de cómo han estudiado la geografía y propuestas para mejorar la enseñanza-aprendizaje de esta disciplina. En M.J. Marrón, M.D. Rosado \& C. Rueda (eds.) Enseñar geografía: La cultura geográfica en la era de la globalización (1st ed., pp. 435-458) Jaén: Grupo de Didáctica de la Geografía de la Asociación de Geógrafos Españoles.

MARRÓN GAITE, M.J. (2011): Educación geográfica y formación del profesorado: Desafíos y perspectivas en el nuevo espacio europeo de educación superior (EEES). Boletín de La Asociación de Geógrafos Españoles, 57, 313-342.

MAYER, F. (2006): Pedagogía comparada. Mexico D.F.: Pax Mexico

MÉNDEZ, F., y MOLINERO, R (1991): Espacios y sociedades. Barcelona: Ariel.

MIRANDA ARREDONDO, P. (2006): Paradigmas dominantes en el proceso de enseñanza aprendizaje de la Geografía: obstáculos epistemológicos para la enseñanza de la ciencia en el siglo XXI. Ponencia presentada en: IV Congreso Iberoamericano de Educación Científica. Consejo Nacional de Ciencia, Tecnología e Innovación Tecnológica. Lima (Perú)

MOLINER, P. (2001): La dynamique des représentations sociales. Grenoble (France): Universitarie de Grenoble.

PÉREZ LIÑAL, A. (2008): El método comparativo: fundamentos y desarrollos recientes. Revista digital de Política comparada, 3, 2-29.

PLANS, P. (1984): Introducción a la Geografía General. Pamplona: Eunsa.

RAYAS, J. (2004): El reconocimiento de las ideas previas como condición necesaria para mejorar las posibilidades de los alumnos en los procesos educativos en ciencias naturales. Revista Xictli de la Unidad UPN 094 D.F. Centro, México. En http://www.unidad094. upn.mx Última revisión 2013/12/20.

REVENTOS, F. (1990): Metodología comparativa y pedagogía comparada. Barcelona: Boixareu.

STODDART, D.R. (1987): To claim de high ground: geography for the end of the century. Transactions, Institute of British Geographers, 12 (3), 327-336.

TULLA, A. (2010): Los nuevos planes de estudio de los títulos de grado en geografía adaptados al modelo del espacio europeo de educación superior (EEES). Estudios Geográficos, 71 (268), 319-338.

UNWIN, T. (1995): El lugar de la Geografía. Madrid: Cátedra.

YÉPEZ HERNÁNDEZ, M. (2010): Representaciones sociales de democracia en estudiantes universitarios de la UAM. Investigación de la comunicación. México: En los albores del siglo XXI. 
